\title{
A Note on Gorenstein Flat Dimension
}

\author{
Driss Bennis \\ Department of Mathematics, Faculty of Science and Technology of Fez, \\ Box 2202, University S. M. Ben Abdellah Fez, Morocco \\ E-mail: driss_bennis@hotmail.com
}

\begin{abstract}
Unlike the Gorenstein projective and injective dimensions, the majority of results on the Gorenstein flat dimension have been established only over Noetherian (or coherent) rings. Naturally, one would like to generalize these results to any associative ring. In this direction, we show that the Gorenstein flat dimension is a refinement of the classical flat dimension over any ring; and we investigate the relations between the Gorenstein projective dimension and the Gorenstein flat dimension.
\end{abstract}

2000 Mathematics Subject Classification: 16E05, 16E10, 16E30

Keywords: Gorenstein flat dimension; flat dimension; copure flat dimension; Gorenstein projective dimension

\section{Introduction}

Throughout this paper, $R$ denotes an associative ring with identity and all modules are unitary. For an $R$-module $M$, we use $\operatorname{id}_{R}(M)$ and $\mathrm{fd}_{R}(M)$ to denote, respectively, the classical injective and flat dimensions of $M$. We use $M^{*}$ to denote the character module $\operatorname{Hom}_{\mathbb{Z}}(M, \mathbb{Q} / \mathbb{Z})$ of $M$.

For every module over an associative ring, Enochs, Jenda, and Torrecillas 9] defined the Gorenstein flat dimension (see Definition 2.1) to complete the analogy between classical homological dimension theory and Gorenstein homological dimension theory. But, they mainly studied it when the base ring is Gorenstein (see also 11]). Their characterization of the Gorenstein flat dimension over Gorenstein rings was generalized, by Chen and Ding [4, to $n$-FC rings (i.e., coherent rings with self-FP-injective dimension at most $n$ ). Also, Christensen gave a characterization of the Gorenstein flat dimension over local Cohen-Macaulay rings with a dualizing module [5, Theorem 5.2.14] (see also [10]). Namely, in Christensen's book, it is shown that there are good results for the Gorenstein flat dimension over Noetherian rings, which are very often local Cohen-Macaulay with a dualizing module. In [12, Holm generalized these results to coherent rings (see also [6]). In this paper, we give some results on the Gorenstein flat dimension that hold over a larger class of rings.

In Section 2, we show that the Gorenstein flat dimension is a refinement of the usual flat dimension over any associative ring, that is Theorem 2.2. Then, in Theorem 2.4. we 
establish a relation between the Gorenstein flat dimension and the copure flat dimension (see Definition 2.3).

In Section 3, we investigate the relations between the Gorenstein flat dimension and the Gorenstein projective dimension. Namely, we establish another situation where the question "Is every Gorenstein projective module Gorenstein flat" has an affirmative answer (see Proposition 3.2). In Theorem 3.3, we show that, if $M$ is an infinitely presented left $R$-module, then $M$ is Gorenstein flat if and only if it is Gorenstein projective.

\section{Gorenstein flat, flat, and copure flat dimensions}

In this section, we investigate the relations between the Gorenstein flat dimension, the flat dimension, and the copure flat dimension.

Recall the definitions of Gorenstein flat modules and Gorenstein flat dimension.

Definition 2.1 ([5]) A complete flat resolution is an exact sequence of flat left $R$-modules,

$$
\mathbf{F}=\cdots \rightarrow F_{1} \rightarrow F_{0} \rightarrow F^{0} \rightarrow F^{1} \rightarrow \cdots,
$$

such that $I \otimes_{R} \mathbf{F}$ is exact for every injective right $R$-module $I$.

$A$ left $R$-module $M$ is called Gorenstein flat ( $G$-flat for short), if there exists a complete flat resolution $\mathbf{F}$ with $M \cong \operatorname{Im}\left(F_{0} \rightarrow F^{0}\right)$.

For a positive integer $n$, we say that a left $R$-module $M$ has Gorenstein flat dimension at most $n$, and we write $\operatorname{Gfd}_{R}(M) \leq n$, if if there exists an exact sequence of $R$-modules $0 \rightarrow G_{n} \rightarrow \cdots \rightarrow G_{0} \rightarrow M \rightarrow 0$, where each $G_{i}$ is Gorenstein flat.

It is well-known that the Gorenstein homological dimensions are, over Noetherian rings, refinements of the classical homological dimensions. In [8] and also in [12, it is proved that the result remains true for the Gorenstein projective and injective dimensions over associative rings. However, in the note after Proposition 3.6 of [1], it is remarked that the Gorenstein flat dimension is a refinement of the classical flat dimension over coherent rings (see also [1, Proposition 3.7 and Corollary 3.8]). Next we show this holds over associative rings.

Theorem 2.2 For a left $R$-module $M, \operatorname{Gfd}_{R}(M) \leq \mathrm{fd}_{R}(M)$ with equality when $\operatorname{fd}_{R}(M)$ is finite.

Proof. The inequality is well-known and follows from the trivial fact that every flat module is Gorenstein flat (see for instance [12, Theorem 3.19]).

Now assume that $\operatorname{fd}_{R}(M)<\infty$. Then, by [14, Lemma 3.51 and Theorem 3.52], $\operatorname{id}_{R}\left(M^{*}\right)=$ $\mathrm{fd}_{R}(M)<\infty$ and so $\operatorname{Gid}_{R}\left(M^{*}\right)=\operatorname{id}_{R}\left(M^{*}\right)$ (from the injective counterpart of [12, Proposition 2.27]). Therefore, combine the last equalities and the inequality $\operatorname{Gid}_{R}\left(M^{*}\right) \leq \operatorname{Gfd}_{R}(M)$ of [12, Theorem 3.11], we get:

$$
\operatorname{fd}_{R}(M)=\operatorname{id}_{R}\left(M^{*}\right)=\operatorname{Gid}_{R}\left(M^{*}\right) \leq \operatorname{Gfd}_{R}(M) .
$$

This gives the desired equality. 
Another dimension which is closely related with the Gorenstein flat dimension is the copure flat dimension, which is defined as follows:

Definition $2.3([7])$ The copure flat dimension of a left $R$-module $M, \operatorname{cfd}_{R}(M)$, is defined to be the largest positive integer $n$ such that $\operatorname{Tor}_{n}^{R}(E, M) \neq 0$ for some injective right $R$ module $E$.

The copure flat dimension is used in the functorial description of the Gorenstein flat dimension as follows [12, Theorem 3.14]: If $R$ is right coherent and $M$ is a left $R$-module with finite Gorenstein flat dimension, then $\operatorname{Gfd}_{R}(M)=\operatorname{cfd}_{R}(M)$. This is a generalization of [4, Corollary 11] and [5, Theorem 5.2.14]. Over any ring we give the following:

Theorem 2.4 For any left $R$-module $M$, we have inequality:

$$
\operatorname{cfd}_{R}(M) \leq \operatorname{Gfd}_{R}(M) .
$$

If $\operatorname{fd}_{R}(M)$ is finite, then we have equalities:

$$
\operatorname{cfd}_{R}(M)=\operatorname{Gfd}_{R}(M)=\mathrm{fd}_{R}(M) .
$$

To prove this we need the following lemma.

Lemma 2.5 Let $M$ be a left $R$-module. If $\mathrm{fd}_{R}(M)<\infty$, then $\operatorname{cfd}_{R}(M)=\mathrm{fd}_{R}(M)$.

Proof. Obviously $\operatorname{cfd}_{R}(M) \leq \mathrm{fd}_{R}(M)$.

For the converse, suppose that $\mathrm{fd}_{R}(M)=n$ for some positive integer $n$. Then there is a right $R$-module $N$ such that $\operatorname{Tor}_{n}^{R}(N, M) \neq 0$, and it follows, by the long exact sequence of Tor, that also $\operatorname{Tor}_{n}^{R}(E(N), M) \neq 0$, where $E(N)$ is the injective envelope of $N$. This implies that $\operatorname{cfd}_{R}(M) \geq n=\mathrm{fd}_{R}(M)$, as desired.

Proof of Theorem 2.4. The inequality $\operatorname{cfd}_{R}(M) \leq \operatorname{Gfd}_{R}(M)$ follows by dimension shifting argument and using the fact that every Gorenstein flat left $R$-module $G$ satisfies $\operatorname{Tor}_{i}^{R}(I, G)=0$ for all $i \geq 1$ and all injective right $R$-modules $I$.

The equalities $\operatorname{cfd}_{R}(M)=\operatorname{Gfd}_{R}(M)=\operatorname{fd}_{R}(M)$ when $\mathrm{fd}_{R}(M)$ is finite follow immediately by the inequalities $\operatorname{cfd}_{R}(M) \leq \operatorname{Gfd}_{R}(M) \leq \operatorname{fd}_{R}(M)$ and Lemma 2.5.

Note that the proof of the equalities of Theorem 2.4 can be used as a proof of the equality of Theorem 2.2 and so the two results can be written in one theorem. Here we separate them because each one has a different aim.

\section{Gorenstein flat and Gorenstein projective dimensions}

In this section, we investigate the relations between the Gorenstein flat dimension and the Gorenstein projective dimension.

First, recall the definitions of Gorenstein projective modules and Gorenstein projective dimension. 
Definition 3.1 ([5]) A complete projective resolution is an exact sequence of projective left $R$-modules,

$$
\mathbf{P}=\cdots \rightarrow P_{1} \rightarrow P_{0} \rightarrow P^{0} \rightarrow P^{1} \rightarrow \cdots,
$$

such that $\operatorname{Hom}_{R}(\mathbf{P}, Q)$ is exact for every projective left $R$-module $Q$.

A left $R$-module $M$ is called Gorenstein projective (G-projective for short), if there exists a complete projective resolution $\mathbf{P}$ with $M \cong \operatorname{Im}\left(P_{0} \rightarrow P^{0}\right)$.

For a positive integer $n$, we say that a left $R$-module $M$ has Gorenstein projective dimension at most $n$, and we write $\operatorname{Gpd}_{R}(M) \leq n$, if there exists an exact sequence of $R$-modules $0 \rightarrow G_{n} \rightarrow \cdots \rightarrow G_{0} \rightarrow M \rightarrow 0$, where each $G_{i}$ is Gorenstein projective.

Motivated by the results in the classical case, there are two principal questions concerning the relations between the Gorenstein projective dimension and the Gorenstein flat dimension of modules. In fact, between the Gorenstein projective modules and the Gorenstein flat modules:

Question A. Is every Gorenstein projective module Gorenstein flat?

Question B. When is a Gorenstein flat module Gorenstein projective?

Several attempts have been made to obtain, as the classical case, an affirmative answer to Question A. In [12, Proposition 3.4], Holm proved, over right coherent ring with finite finitistic projective dimension, that every Gorenstein projective left module is Gorenstein flat. In fact, this result holds, by the proof of [12, Proposition 3.4], over right coherent rings such that every flat left module has finite projective dimension, which is remarked in 6, Proposition 3.7]. Here we give the following result:

Proposition 3.2 If every injective right $R$-module has finite flat dimension, then $\operatorname{Gfd}_{R}(M) \leq$ $\operatorname{Gpd}_{R}(M)$ for every left $R$-module $M$.

Proof. To prove the inequality, it is sufficient to prove that every Gorenstein projective left module is Gorenstein flat, which is equivalent to proving that every complete projective resolution is complete flat.

Consider then a complete projective resolution $\mathbf{P}$ and an injective right $R$-module $I$. By hypothesis, $\operatorname{fd}_{R}(I)=n$ for some positive integer $n$. Then, we have an exact sequence

$$
0 \rightarrow F_{n} \rightarrow \cdots \rightarrow F_{0} \rightarrow I \rightarrow 0
$$

where each $F_{i}$ is a flat right $R$-module. Let $I_{i}=\operatorname{Im}\left(F_{i} \rightarrow F_{i-1}\right)$ for $1 \leq i \leq n-1$ and $I_{0}=I$.

Consider the short exact sequence

$$
0 \rightarrow F_{n} \rightarrow F_{n-1} \rightarrow I_{n-1} \rightarrow 0
$$

Then,

$$
0 \rightarrow F_{n} \otimes_{R} \mathbf{P} \rightarrow F_{n-1} \otimes_{R} \mathbf{P} \rightarrow I_{n-1} \otimes_{R} \mathbf{P} \rightarrow 0
$$


is a short exact sequence of complexes. Since $F_{n}$ and $F_{n-1}$ are flat, $F_{n} \otimes_{R} \mathbf{P}$ and $F_{n-1} \otimes_{R} \mathbf{P}$ are exact, so is $I_{n-1} \otimes_{R} \mathbf{P}$.

Now consider the short exact sequence

$$
0 \rightarrow I_{n-1} \rightarrow F_{n-2} \rightarrow I_{n-2} \rightarrow 0 .
$$

Then,

$$
0 \rightarrow I_{n-1} \otimes_{R} \mathbf{P} \rightarrow F_{n-2} \otimes_{R} \mathbf{P} \rightarrow I_{n-2} \otimes_{R} \mathbf{P} \rightarrow 0
$$

is a short exact sequence of complexes. Since $F_{n-2}$ is flat and by the argument above, $I_{n-1} \otimes_{R} \mathbf{P}$ and $F_{n-2} \otimes_{R} \mathbf{P}$ are exact, so is $I_{n-2} \otimes_{R} \mathbf{P}$.

The argument above can be applied successively until we conclude that the sequence $I \otimes_{R} \mathbf{P}$ is exact. This implies the desired result.

Note that the rings that satisfy the condition of Proposition 3.2 were investigated in $[3]$.

Now, we investigate Question B. In [1, Question B was investigated for a particular case of Gorenstein projective and flat modules, such that well-known results in the classical case were extended (please see [1, Propositions 3.9 and 3.12 and Corollary 3.10]). Here, we give a situation where a Gorenstein flat module is Gorenstein projective.

It is well-known that a finitely presented module is flat if and only if it is projective. In Gorenstein homological dimension theory, there is an analogous (in fact a generalization) of this result over Noetherian rings [5, Theorem 5.1.11]. Namely, it is proved, over Noetherian rings, that a finitely generated module is Gorenstein flat if and only if it is Gorenstein projective. In [1, Proposition 1.3], it is remarked that [5. Theorem 5.1.11] can be generalized to coherent rings and for finitely presented modules. Next we generalize this by showing, over associative rings, that the same equivalence holds for infinitely presented modules.

Recall that a left $R$-module $M$ is said to be infinitely presented, if it admits a free resolution

$$
\cdots \rightarrow F_{1} \rightarrow F_{0} \rightarrow M \rightarrow 0
$$

such that each $F_{i}$ is a finitely generated free left $R$-module. For instance, over a left Noetherian ring, every finitely generated left module is infinitely presented; and generally, over a left coherent ring, every finitely presented left module is infinitely presented.

Theorem 3.3 Let $M$ be an infinitely presented left $R$-module. Then, $M$ is Gorenstein flat if and only if it is Gorenstein projective.

The proof of this theorem involves the following lemma which generalizes [5, Lemma 5.1.10].

Recall that an exact sequence of finitely generated free left $R$-modules $\mathbf{L}$ is called a complete resolution by finitely generated free left $R$-modules, if the dual complex $\operatorname{Hom}_{R}(\mathbf{L}, R)$ is exact [5, Definition 4.1.2].

Lemma 3.4 Let $\mathbf{L}$ be an exact sequence of finitely generated free left $R$-modules. The following are equivalent: 
1. $\mathbf{L}$ is a complete resolution by finitely generated free left $R$-modules;

2. $\mathbf{L}$ is a complete projective resolution;

3. $\mathbf{L}$ is a complete flat resolution.

Proof. First, using [14, Lemma 3.59], the equivalence (1) $\Leftrightarrow(3)$ has the same proof as the that of $(i) \Leftrightarrow($ iii) of [5, Lemma 5.1.10] (see errata on the Christensen's homepage: http://www.math.ttu.edu/ lchriste/).

Now, since (2) is stronger than (1), it remains to prove the implication (1) $\Rightarrow(2)$. Let

$$
\mathbf{L}=\cdots \rightarrow L_{1} \rightarrow L_{0} \rightarrow L_{-1} \rightarrow L_{-2} \rightarrow \cdots
$$

be a complete resolution by finitely generated free left $R$-modules. We decompose $\mathbf{L}$ into short exact sequences

$$
\left(\alpha_{i}\right)=\quad 0 \rightarrow N_{i+1} \rightarrow L_{i} \rightarrow N_{i} \rightarrow 0,
$$

where $N_{i}=\operatorname{Im}\left(L_{i} \rightarrow L_{i-1}\right)$ for $i \in \mathbb{Z}$. To prove that $\mathbf{L}$ is a complete projective resolution, it is sufficient to prove, for every $i \in \mathbb{Z}$, that $\operatorname{Ext}_{R}^{1}\left(N_{i}, Q\right)=0$ for every projective left $R$-module $Q$. Indeed, if such condition holds, then the sequences

$$
0 \rightarrow \operatorname{Hom}\left(N_{i}, Q\right) \rightarrow \operatorname{Hom}\left(L_{i}, Q\right) \rightarrow \operatorname{Hom}\left(N_{i+1}, Q\right) \rightarrow 0
$$

are all exact for every projective left $R$-module $Q$. This implies, by assembling the sequences $\left(\alpha_{i}\right)$, that $\mathbf{L}$ is a complete projective resolution.

Then, we prove, for every $i \in \mathbb{Z}$, that $\operatorname{Ext}_{R}^{1}\left(N_{i}, Q\right)=0$ for every projective left $R$-module $Q$. Note first that each $R$-module $N_{i}$ is infinitely presented and satisfies $\operatorname{Ext}_{R}^{n}\left(N_{i}, R\right)=0$ for all $n>0$.

By Lazard's Theorem [2, $\S 1, \mathrm{~N}^{\circ} 6$, Theorem 1], there exists, for every flat left $R$-module $F$, a direct system $\left(F_{j}\right)_{j \in J}$ of finitely generated free left $R$-modules over a directed index set $J$ such that $\underset{\lim }{\longrightarrow} F_{j} \cong F$. From [2, Exercise 3, p. 187] (or similarly to the proof of [8, Lemma 3.1.16]), we get, for every $i \in \mathbb{Z}$,

$$
\begin{aligned}
\operatorname{Ext}_{R}^{1}\left(N_{i}, F\right) & \cong \operatorname{Ext}_{R}^{1}\left(N_{i}, \underline{\lim } F_{j}\right) \\
& \cong \underline{\lim } \operatorname{Ext}_{R}^{1}\left(N_{i}, F_{j}\right) .
\end{aligned}
$$

Now, since every direct sum is the direct limit of its finite partial sum ordered by inclusion, [2, Exercise 3, p. 187] implies also that $\operatorname{Ext}_{R}^{1}\left(N_{i}, F_{j}\right)=0$. Therefore, for every projective (then flat) left $R$-module $Q, \operatorname{Ext}_{R}^{1}\left(N_{i}, Q\right)=0$, which completes the proof.

Proof of Theorem 3.3. Using Lemma 3.4 above, the "if" part is proved along the same lines as [5, Theorem 4.2.6] and the "only if" part is proved similarly to [5, Theorem 5.1.11].

There are examples, over Noetherian rings, of finitely generated (then infinitely presented) modules which are Gorenstein projective (then Gorenstein flat) but they are not projective (then not flat) (see for instance [5. Examples 1.1.13 and 4.1.5]). Namely, any ideal of a 1-Gorenstein ring (i.e., Noetherian with self-injective dimension at most 1) is infinitely presented and Gorenstein projective. 
We take advantage of Lemma 3.4 and its proof to give a result for the Gorenstein projective dimension. Precisely, using the proof of Lemma 3.4, we easily deduce the following characterization of Gorenstein projective dimension of an infinitely presented left module. This generalizes [5, Theorem 1.2.7] (see also [5, Theorem 4.4.12 and Corollary 4.4.13]).

Corollary 3.5 Let $M$ be an infinitely presented left $R$-module with finite Gorenstein projective dimension and let $n \geq 0$ be a positive integer. Then, the following are equivalent:

1. $\operatorname{Gpd}_{R}(M) \leq n$;

2. $\operatorname{Ext}_{R}^{i}(M, F)=0$ for all $i>n$ and all flat left $R$-modules $F$;

3. $\operatorname{Ext}_{R}^{i}(M, F)=0$ for all $i>n$ and all left $R$-modules $F$ of finite flat dimension;

4. $\operatorname{Ext}_{R}^{i}(M, R)=0$ for all $i>n$.

Consequently, the Gorenstein projective dimension of $M$ is also determined by the formulas:

$$
\begin{aligned}
\operatorname{Gpd}_{R}(M) & =\sup \left\{i \in \mathbb{N} \mid \operatorname{Ext}_{R}^{i}(M, F) \neq 0 \text { for some flat left } R \text {-module } F\right\} \\
& =\sup \left\{i \in \mathbb{N} \mid \operatorname{Ext}_{R}^{i}(M, F) \neq 0 \text { for some left } R \text {-module } F \text { with } \operatorname{fd}_{R}(F)<\infty\right\} \\
& =\sup \left\{i \in \mathbb{N} \mid \operatorname{Ext}_{R}^{i}(M, R) \neq 0\right\} .
\end{aligned}
$$

It is important to note that the condition " $M$ has finite Gorenstein projective dimension" in Corollary 3.5 above can not be dropped. Indeed, Jorgensen and Şega 13, Theorem 1.7] constructed, over an artinian ring $R$, a finitely generated (then infinitely presented) $R$-module $M$ such that $\operatorname{Gpd}_{R}(M)=\infty$ and $\operatorname{Ext}_{R}^{i}(M, R)=0$ for all $i>0$.

Acknowledgements. The author thanks the referee for very helpful comments and suggestions.

\section{References}

[1] D. Bennis and N. Mahdou, Strongly Gorenstein projective, injective, and flat modules, J. Pure Appl. Algebra 210 (2007) 437-445.

[2] N. Bourbaki, Algèbre Homologique, Chapitre 10, Masson, Paris, 1980.

[3] J. Chen and N. Ding, The flat dimensions of injective modules, Manuscripta Math. 78 (1993) $165-177$.

[4] J. Chen and N. Ding, Coherent rings with finite self-FP-injective dimension, Comm. Algebra 24 (1996) 2963-2980.

[5] L. W. Christensen, Gorenstein dimensions, Lecture Notes in Math., 1747, Springer, Berlin, 2000.

[6] L. W. Christensen, A. Frankild, and H. Holm, On Gorenstein projective, injective and flat dimensions - a functorial description with applications, J. Algebra 302 (2006) $231-279$. 
[7] E. E. Enochs and O. M. G. Jenda, Copure injective resolutions, flat resolvents and dimensions, Comment. Math. Univ. Carolin. 34 (1993) 203-211.

[8] E. E. Enochs and O. M. G. Jenda, Relative homological algebra, de Gruyter Expositions in Mathematics, vol. 30, Walter de Gruyter \& Co., Berlin, 2000.

[9] E. E. Enochs, O. M. G. Jenda, and B. Torrecillas, Gorenstein flat modules, Nanjing Daxue Xuebao Shuxue Bannian Kan 10 (1993) 1-9.

[10] E. E. Enochs, O. M. G. Jenda, and J. Xu, Foxby duality and Gorenstein injective and projective modules, Trans. Amer. Math. Soc. 348 (1996) 3223-3234.

[11] E. Enochs and J. Xu, Gorenstein Flat Covers of Modules over Gorenstein Rings, J. Algebra 181 (1996) 288-313.

[12] H. Holm, Gorenstein homological dimensions, J. Pure Appl. Algebra 189 (2004) 167193.

[13] David A. Jorgensen and Liana M. Şega, Independence of the total reflexivity conditions for modules, Algebr. Represent. Theory 9 (2006) 217-226.

[14] J. Rotman, An Introduction to Homological Algebra, Pure and Applied Mathematics, 85, Academic Press, New York-London, 1979. 